\title{
Suitability of Shape and Texture Features in Retrieval of Medicinal Plants' Images in Indian Context
}

\author{
Basavaraj S. Anami, Suvarna S. Nandyal, and A. Govardhan
}

\begin{abstract}
Content Based Image Retrieval (CBIR) is important in computer aided plant species recognition. Texture and shape information have been the primitive image descriptors in content based image retrieval systems. This paper presents a novel framework for combining both the features, texture and shape information, and achieve higher retrieval efficiency. The study provides a methodology for retrieving medicinal plants images from a database of medicinal plant images based on shape and texture features. The shape descriptors include Zernike moment, Fourier descriptor (FD), Generic fourier Descriptor(GFD) and for texture descriptors gabor filters are used. The similarity measures, euclidean distance of each medicinal plant image from the database to query image is used. The images are sorted based on similarity of Euclidean distance. The retrieval experiments are carried on different training and test medicinal plant images. The effectiveness of different descriptors is confirmed by the experimental results. We have investigated shape and texture features for medicinal plant retrieval by successively combining the different transforms. The retrieval efficiency is reported through precision and recall rate. Experimental results by combining Gabor and Zernike transform outperforms the all other methods.
\end{abstract}

Index Terms-CBIR, Fourier descriptor, medicinal plant, zernike moments.

\section{INTRODUCTION}

Medicinal Plant species identification is a process of assignment of each individual plant to a descending series of groups of related plants ad judged by common characteristics. These medicinal plants are used in ayurveda. Hence, it is important and essential to quickly recognize and identify the medicinal plant species on the earth. Ayurveda is being accepted worldwide as an alternate medicine to allopathic medicine. Hence, a database of medicinal plant species (Herbs) is essential for any ayurvedic practitioner for preparation of herbal medicine. Herbal plants have global market. This is the proper time for India to make relentless efforts to take substantial cultivation and share in current herbal market. The need for developing countries to acquire technologies and techniques for programmed cultivation of medicinal plants is a current issue.Hence, in this paper we have proposed different methods for medicinal plant image retrieval and efficiency of these methods are reported through

Manuscript received July 27, 2012; revised September 10, 2012.

Basavaraj S. Anam is with the K.L.E. Institute of Technology, HUBLI, and Karnataka, India (e-mail:anami_basu@hotmail.com)

Suvarna S Nandyal is with the JNTU Hyderabad, AP, India and Dept of CSE,P.D.A.College of engg, GULBARGA, Karnataka, India (e-mail:suvarna_nandyal@yahoo.com)

A. Govardhan is with the JNTU Hyderabad, Andhra Pradesh, India (e-mail:govardhan_cse@yahoo.co.in the graph.

To greatly speedup the process of medicinal plant species identification, collection, and monitoring, ayurveda practioners need to have the world's herbaria at their fingertips. There is a critical shortfall in identifying different natural databases. Finding a species quickly requires that the user know in advance the name of the species. Computer vision algorithms can remove this obstacle, allowing a user to search through this data using algorithms that match images of newly collected specimen with images of those previously discovered and described. Hence, this is the motivation for the present work on medicinal plant retrieval based on shape and texture descriptors. In this paper a medicinal plant image is compared to all possible matches and in matter of seconds the user is shown information about the best matching species in sorted order.

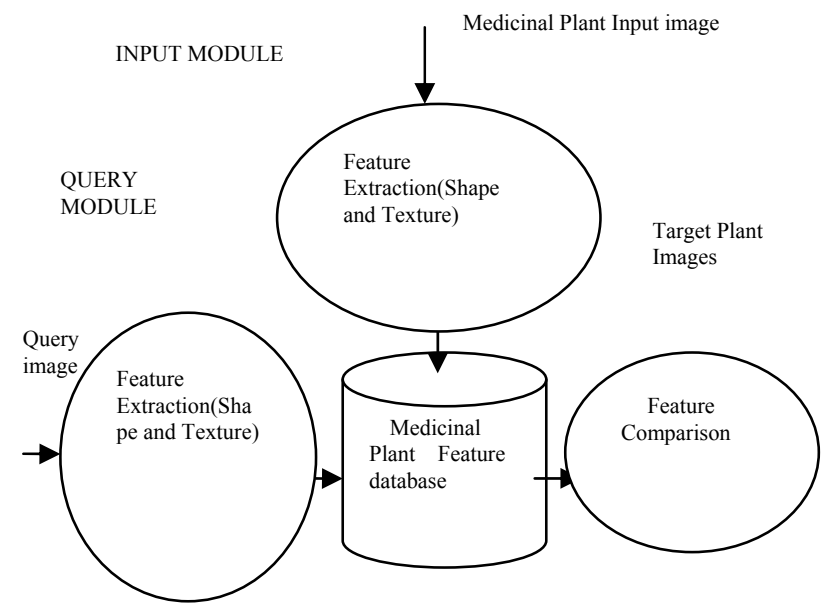

Fig. 1. Block diagram of content based image retrieval system

Fig. 1 shows block diagram of Content Based Image Retrieval System for medicinal plant database. It consists of three main modules input module, query module, and retrieval module. In the input module, the feature vector is extracted from the input plant images and the feature vector of each plant image is stored in the database. While in the query module, a medicinal plant query image is inputted, and its feature vector is extracted for the latter retrieval using shape and texture feature extraction techniques. During the retrieval process, the feature vector of the query image is compared with each vector in the database, and the corresponding target (similar) plant images are outputted.

The paper is organized into five sections. In section 2 literature survey is presented. Section 3 deals with shape and texture feature extraction techniques. We present comparative experimental results of medicinal plant images by using different descriptors in section 4 . The retrieval efficiencies have been demonstrated by using recall and precision graphs in the Appendix section. Section 5 
concludes the paper with a discussion.

\section{LITERATURE SURVEY}

In India, the medicinal plants are deterioting day by day. Hence the first step of protecting the medicinal plants is to automatically recognize or classify them, that is understand what they are and where they come from and what for they are used. But it is very difficult for one to recognize a plant in hand correctly and immediately because there are so many kinds of medicinal plants unknown to us on the earth. So we wish to use image processing and pattern recognition methods to retrieve medicinal plant. In order to retrieve an image from a database an efficient feature extraction technique has to be used. Hence a brief literature survey about shape and texture feature extraction technique is presented.

Several methods for shape representation have been developed. The shape description techniques can be divided into two types, boundary based and region based techniques. The region based methods consider the whole area of the object whereas the boundary based methods concentrate merely on object boundary line. In this paper we consider the boundary based methods for medicinal plant shape classification. The most common boundary based shape descriptors are chain codes and Fourier descriptors in multiple resolution. During recent years curvature scale space (CSS) shape representation is used [9] for image corner detection.Dengsheng Zhang et al[4] made a comparative study of fourier descriptors for shape representation using different fourier invarients. The study shows that among shape signatures complex coordinates, centroid distance is the best shape signature for shape retrieval. But these shape descriptors are undesirable for generic shape descriptors. Generic fourier descriptor has been used to overcome the drawbacks of existing shape representation techniques [6]. These feature vectors are given as input to conent based image retrieval system. Here we briefly outline the efficiency analysis some of the shape and texture feature extraction techniques used in content based image retrieval.

Andre Folkers,Hanan Samet et al[3] has proposed a system that enables the pictorial specification of queries in an image database is described. The queries are comprised of rectangle, polygon, ellipse, and B-splineshapes. The queries specify which shapes should appear in the target image as well as spatial constraints on the distance between them and their relative position. The retrieval process makes use of the contour of the shape using Fourier descriptors. These are used in a system to locate logos in an image database. They have showed that fourier descriptor is an efficient shape extraction technique for arbitrary shapes.

Contour-based shape feature extraction carried out on gray scale medical images. The fourier descriptior method with brightness of boundary pixels is used to locate the shape of the images. Fourier transforms [10] are used for the shape signatures to compute Fourier coefficients, and standardized brightness is used as weight. Fourier descriptors are invariant to translation, scaling, rotation and change of start point. It is found that the Fourier descriptor with brightness gave a better performance than the usual fourier descriptor.Iivari Kunttu,Leena Lepisto et al[11], has made a comparison between Fourier-based descriptor, multiscale fourier descriptor and (CSS) Curvature Scale Space, for shape characterization in CBIRl. Fourier descriptor is combined with wavelet transform. The multiscale Fourier descriptor is formed by applying the complex wavelet transform to the boundary function. In this comparision fourier descriptors proved best in the classification of different shapes.In the recent study [8] Zhang and Lu, fourier descriptors gave better retrieval results in image retrieval than CSS-representation.

For CBIR purpose a shape descriptor should be affine, invariant, compact and easy to derive and match. In terms of these, Zernike moments [14] are the one which gives a more robust to changes caused by image rotation, translation and scaling. Zernike moments have been used on MPEG-7 region shape DB CE-2 database which consists of 3621 binary shapes of trademarks. Experiment results show the robustness of the Zernike moments under various image transformations and its superior performance in noise robustness. Ye Bin, Peng Jia-xiong [16] have shown that image retrieval on image database of device-mark type trademark images, MPEG-7database, Zernike moment outperforms other region based descriptor.

A variety of techniques ranging from statistical methods to multiresolution filtering technique is useful for texture analysis. Two dimensional gabor filter is proved to be very useful texture analysis and is widely used in literature.X.Fu and Y.Li et al [14] have demonstrated gabor filter(GF) and zernike moments $(Z M)$ feature extraction technique on face, fingerprint and MPEG-7 database. ZM is effective for face and MPEG-7 database.GF is effective for face and fingerprint database but weak for MPEG-7 shape database. They achieved best retrieval rate for each database combining both the technique.Atul Sajjanhar, Guojun Lu et al[1], has performed image retrieval using generic fourier descriptor on MPEG-7 still images Content set and found effective retrieval efficiency.In this paper we apply fourier transform[11] for the retrieval of different medicinal plants. In addition to shape representation, texture features are also used for content based medicinal plant image retrieval. We combined gabor texture features[7] and zernike features[14].

\section{FEATURE EXTRACTION}

Human vision system recognizes a plant based on its visual appearance. Botonist use plant morphology (or phytomorphology) as the general term for the study of the morphological characteristics of plants. It deals with physical form and external structure of plants. A wide variety of plant images is contains heterogeneous texture in stem and leafy parts and varies in shape and size. Hence a robust shape and texture feature has to be used for recognition and retrieval of plants. This section presents the medicinal plants image data base collection and feature extraction techniques.

\section{A. Medicinal Plants Image Database}

The medicinal plants database used in our experiment is collected by our-self from different herbarium and fields. The experiment results have been implemented with this database which includes 100 pictures formed by ten image categories. Fig. 2 depicts a sample of each category. The images are 
stored in JPEG format with 256x256.

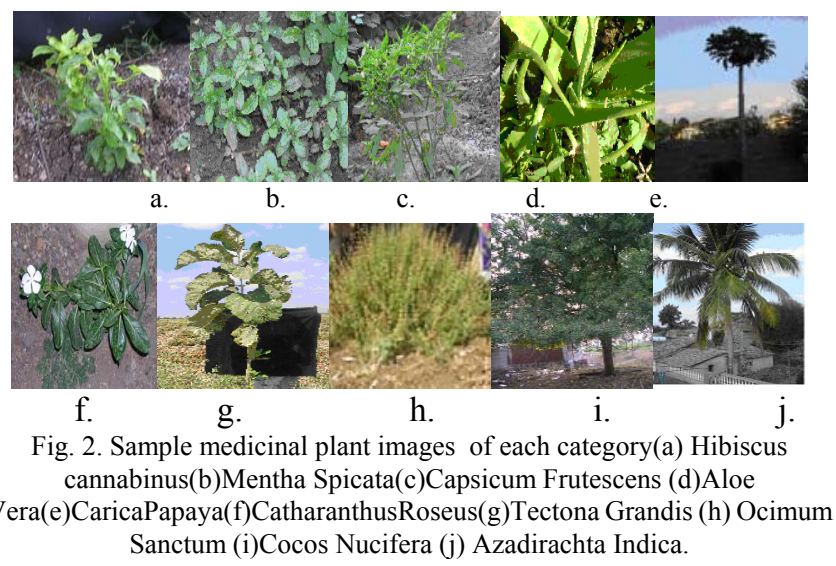

B. Shape Based Feature Extraction

1) Pre-processing

Image

Plant
Binarisation $\rightarrow$ Denoising $\rightarrow \begin{aligned} & \text { Boundary } \\ & \text { Connection }\end{aligned} \rightarrow \begin{gathered}\text { Boundry } \\ \text { Tracing }\end{gathered}$

Fig. 3. Preprocessing of medicinal plant image

The shapes we consider in this paper are medicinal plant images outline shapes which are described as single plane closed curves. The shapes in our database are plants images in the form of gray level images. The preprocessing is to extract the boundary information or coordinates of the boundary from the shape. The block diagram for preprocessing is shown in Fig. 3.The first step in the preprocessing is to convert the plant image into binary image by using a simple threshold.In reality, plant images are often corrupted with noise, as a result, the plant obtained after threshold usually has noise around the shape boundary, therefore, a denoise process is applied. The denoising process eliminates those isolated pixels and those isolated small regions or segments.A $m$-connectivity technique is used to fill the gaps between boundary points. The plant shape is traced using a 8- connectivity contour tracing technique to obtain the plant boundary coordinates as shown in Fig. 4(c).
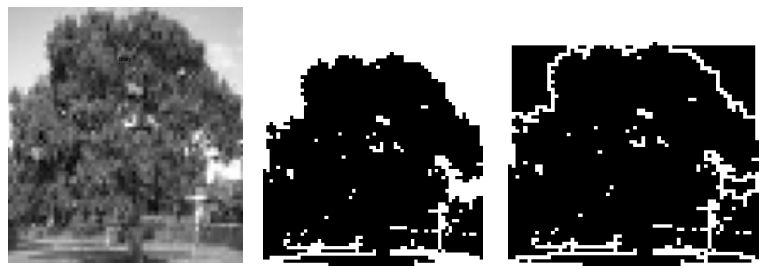

Fig. 4. (a)Binaryimage (b) Denoised

(c)Traced image

\section{2) Fourier descriptor}

Fourier transformation on shape signatures is used for shape analysis for medicinal plants. The Fourier transformed coefficients form the fourier descriptors of the shape. These descriptors represent the shape of the medicinal plant in a frequency domain. The lower frequency descriptors contain information about the general features of the plant shape, and the higher frequency descriptors contain information about finer details of the plant such as leaflets and branch pattern.

Algorithm 1: Feature extraction using Fourier descriptor. Input: Original RGB color plant image.

Output: Shape signature, complex coordinates, centroid distance,boundary points and angle.

Start:

Step 1. Read input medicinal plant image and apply preprocessing and resize it to $64 \times 64$.

Step 2. The $\mathrm{x}(\mathrm{t}), \mathrm{y}(\mathrm{t})$ coordinates of the boundary coordinates are computed, as $s(t)$ whereas $t=0$ to maximum length(L) of boundary coordinate.

Step 3. The centroid of the plant image is calculated using equation

$$
x_{c}=\frac{1}{L} \sum_{t=0}^{L-1} x(t), \quad y_{c}=\frac{1}{L} \sum_{t=0}^{L-1} y(t)
$$

Step 4. With $\mathrm{N}=512$ sample points, the shape signature $\mathrm{s}(\mathrm{t}), \mathrm{t}=0,1, \ldots, L$, assuming it is normalized to $N$ points in the sampling stage, the discrete Fourier transform of $s(t)$ is calculated using equation

$$
\mathrm{U}_{\mathrm{n}}=\frac{1}{\mathrm{~N}} \sum \mathrm{s}(\mathrm{t}) \exp \left(\frac{-\mathrm{j} 2 \mathrm{n} \pi}{\mathrm{N}}\right), \quad \mathrm{n}=0,1, \ldots \ldots \mathrm{N}-1 .
$$

The coefficients $u_{n}, n=0,1, \ldots, N-1$, are called Fourier descriptors (FD) of the shape, denoted as $\quad \mathrm{FD}_{\mathrm{n},} n=0,1, \ldots$, $N-1$.

Step 5. Compute complex coordinates from the set of boundary coordinates using equation (3)

$$
z(t)=x(t)+i y(t)
$$

In order to eliminate the effect of bias, we have used the shifted coordinates function:

$$
z(t)=\left[x(t)-x_{c}\right]+i\left[y(t)-y_{c}\right]
$$

where $\left(\mathrm{x}_{\mathrm{c},} \mathrm{y}_{\mathrm{c}}\right)$ is the centroid of the medicinal plant, which is the average of the plant boundry coordinates. This shift makes the medicinal plant shape representation invariant to translation.

Step 6. Centroid distance $\mathrm{r}(\mathrm{t})$ is obtained from $(x c, y c)$ of plant image and is given by equation (5)

$$
r(t)=\left(\left[x(t)-x_{c}\right]^{2}+\left[y(t)-y_{c}\right]^{2}\right)^{1 / 2}
$$

Step 7. For the set of boundary points $[x(t), y(t)], t=0 \ldots L$ curvature signature is calculated.

Step 8. The boundary angle $\theta(t)$ is found interms of radians. Then, the difference between two angles gives the curvature angle between successive plant boundry points and is given by equation(6)

$\mathrm{K}(\mathrm{t})=\theta(\mathrm{t})-\theta(\mathrm{t}-1)$ Where $\theta(\mathrm{t})=\arctan \frac{\mathrm{y}(\mathrm{t})-\mathrm{y}(\mathrm{t}-1)}{\mathrm{x}(\mathrm{t})-\mathrm{x}(\mathrm{t}-\mathrm{w})}$

where $\mathrm{w}$ is window size between $0.2 \pi$.

where $\theta(t)$ is defined as above.

Step 9. The cumulative angular function $\varphi(t)$, the net amount of angular bend between the starting position $z(0)$ and position $z(t)$ on the medicinal plant boundary is calculated using equation (7).

$$
\varphi(\mathrm{t})=[\theta(t)-\theta(0)] \bmod (2 \pi)
$$


Stop.

3) Generic-fourier descriptor (GFD)

Generic Fourier Descriptor is used [6] to extract features from spectral domain by applying 2-D Fourier transform (FT) on polar raster medicinal plant image. Shape analysis using FT is backed by well developed and well understood Fourier theory. However, it is not desirable to acquire shape features using FT directly, because the acquired features are not rotation invariant. Therefore, a modified polar fourier transform(MPFT) is proposed by treating the polar image in polar space as a normal two-dimensional rectangular image in Cartesian space. Consequently, for a given medicinal plant image $f(x, y)$, the MPFT is defined as in equation (8)

density $B$ or magnetic field strength symbolized as $\mu_{0} H$. Use the center dot to separate compound units, e.g., "A $\cdot \mathrm{m}^{2}$."

$$
\operatorname{PF}(\rho, \varphi)=\sum_{\mathrm{r} \text { i }} \mathrm{f}\left(\mathrm{r}, \theta_{\mathrm{i}}\right) \exp \left[\mathrm{j} 2 \pi\left(\frac{\mathrm{r}}{\mathrm{R}} \rho+\frac{(2 \pi) \mathrm{i}}{\mathrm{T}} \varphi\right)\right]
$$

where

$0 \leq \mathrm{r}=\left[\left(\mathrm{x}-\mathrm{x}_{\mathrm{c}}\right)^{2}+\left(\mathrm{y}-\mathrm{y}_{\mathrm{c}}\right)^{2}\right]^{1 / 2}<\mathrm{R}$ and $\quad \theta_{\mathrm{i}}=\mathrm{i}(2 \pi / \mathrm{T})(0 \leq \mathrm{i}<\mathrm{T}) ;$ $\left(\mathrm{x}_{\mathrm{c}}, \mathrm{y}_{\mathrm{c}}\right)$ is the centre of mass of shape, $0 \leq \rho<\mathrm{R}, 0 \leq \varphi<\mathrm{T}$.

$R$ is the radius of the plant image in polar space and T is angular resolutions varying between 0 to 360 . The physical meanings of $\rho$ and $\varphi$ are clear; they are the pth radial frequency and the $\varphi$ th angular frequency respectively. The determination of the number of $\rho$ and $\varphi$ for shape description is physically achievable, because shape features are normally captured by the few lower frequencies. For medicinal plant image we have used $0<\rho<=3$ and $0<\varphi<=9$.

Algorithm 2: Feature extraction using GFD:

Input: Original RGB color input plant image

Output: 36 GFD feature vectors.

\section{Start:}

Step 1: Read the input image $\mathrm{I}(\mathrm{x}, \mathrm{y})$ and apply the preprocessing and resize it to $64 \times 64$.

Step 2: Compute the fourier transform of grayscale image call it as $\mathrm{f}(\mathrm{x}, \mathrm{y})$.

Step 3: Convert the fourier transform $\mathrm{f}(\mathrm{x}, \mathrm{y})$ image to polar coordinate space using the equation $\mathrm{x} * \cos \theta+\mathrm{y} * \sin$ $\theta$, where as $0<\theta<2 \pi$.Call it as $\mathrm{f}(\mathrm{r}, \theta)$

Step 4: Calculate the generic fourier descriptor feature vector values using equation (8).

\section{Stop.}

Fig. 5(a) shows the Calotropis gigantea medicinal plant image from database and its side view is shown in bottom row. If 2-DFT is applied on these rectangular FFT image, the polar FT has the similar form to the conventional 2-D discrete FT in Cartesian space. It can be observed from Fig. 5(c) that rotation of pattern in Cartesian space results in circular shift in polar space. The circular shift does not change the spectra distribution on polar space. This is particularly well-suited for plant shape representation, because for efficient shape representation, the number of spectra features selected to describe the shape should not be large. The acquired polar Fourier coefficients are translation and rotation invariant. For efficient shape description, only a small number of GFD features are selected for shape representation. In our implementation, 36 GFD features reflecting 4 radial frequencies and 9 angular frequencies are selected to index the shape. The selected GFD features form a feature vector which is used for indexing the shape.
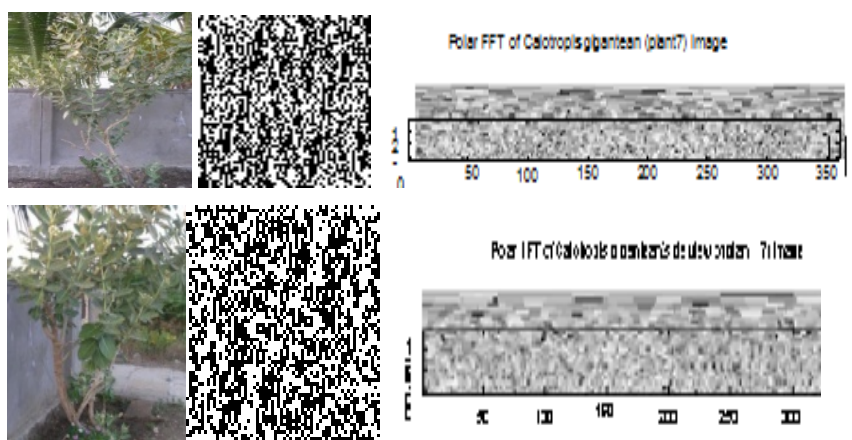

(a)

(b)

(c)

Fig 5.(a)Calotropis gigantea image(Plant7-Top row)Front view,sideview(bottom)(b) FFT of Plant7 and Plant 10 image (c)Polar image plotted into Cartesian space

\section{Zernike Moments Feature Extraction}

Zernike moments (ZM)[14] are one of the most popular shape descriptors and have many desirable properties, such as rotation invariance, robustness to noise, expression efficiency. Hence in this paper we have used Zernike moments for Helpful Hints.extracting the plant shape with $n$ $=4$ (moment order) and $\mathrm{m}=4$ (repetition). The complex ZM are derived from Zernike polynomials which are a set of complex, orthogonal polynomials defined over the interior of a unit circle $x^{2}+y^{2}=1$

$$
\begin{aligned}
& \mathrm{V}_{\mathrm{nm}}(\mathrm{x}, \mathrm{y})=\mathrm{V}_{\mathrm{nm}}(, \theta)=\mathrm{R}_{\mathrm{nm}}(\rho) \exp (\mathrm{jm}) \\
& \text { where } \rho=\sqrt{\left(\mathrm{x}^{2}+\mathrm{y}^{2}\right)} \theta=\operatorname{atan}\left(\frac{\mathrm{y}}{\mathrm{x}}\right)
\end{aligned}
$$

$$
\begin{gathered}
A_{n m}=\frac{n+1}{n} \sum_{x} \sum_{y} f(x, y) V_{n m}(x, y), x^{2}+y^{2} \leq 1 \\
R_{n m}(\rho)=\sum_{s=0}^{\frac{n-\mid m m}{2}}(-1)^{s} \frac{(n-s) !}{s !\left(\frac{n-|m|}{2}-s\right) !\left(\frac{n-|m|}{2}-s\right) !} \rho^{n-2 s}
\end{gathered}
$$

It has been shown that [2] the ZM on a rotated image have the same magnitudes. Therefore $\left|\mathrm{A}_{\mathrm{nm}}\right|$ is used as a rotation invariant feature of the image function. ZM feature vector with moment order 4 and repetition 1 , is given by $\mathrm{f}=[|\mathrm{A} 00||\mathrm{A} 01| \ldots .|\mathrm{A} 14|] ;$ This feature vector is normalized to $[0,1]$ range by $\mathrm{z}$-score normalization

$$
\mathrm{f}_{\text {Zernike }}=\frac{\mathrm{f}-\mu}{\sigma}
$$

where $\mu$ is the mean and $\sigma$ is the standard deviation

Algorithm 3: Feature extraction using Zernike Moments Input: Original RGB color plant image

Output: Zernike shape feature values.

\section{Start:}

Step 1: Read input image and apply preprocessing.

Step 2: Calculate the zernike polynomials $\rho, \theta$ and $\mathrm{V}_{\mathrm{nm}}$ using equation (9). 
Step 3: Eleven zernike feature values are calculated for $\mathrm{n}=4$ and $\mathrm{m}=4$.

$$
\mathrm{f}=\left[\mathrm{A}_{00}, \mathrm{~A}_{01}, \mathrm{~A}_{10}, \mathrm{~A}_{12}, \mathrm{~A}_{21}, \mathrm{~A}_{31}, \mathrm{~A}_{13}, \mathrm{~A}_{23}, \mathrm{~A}_{32}, \mathrm{~A}_{41}, \mathrm{~A}_{14}\right] .
$$
'f'.

Step 4: Calulate the mean and standard deviation value of

Step 5: Find the normalized zernike feature values with the equation (12).

Stop.

\section{Texture Based Feature Extraction}

Texture, gives a global shape feature which is used to associate related shapes. Here we have used the Gabor filters for texture features.

\section{Gabor filters (GF)}

Basically, Gabor filters are a group of wavelets, with each wavelet capturing energy at a specific frequency and a specific direction [14]. Expanding a signal using this basis provides a localized frequency description, therefore capturing local features/energy of the signal. Texture features is extracted from this group of energy distribution. A 2D Gabor function $\mathrm{g}(\mathrm{x}, \mathrm{y})$ and its Fourier transform $\mathrm{G}(\mathrm{u}, \mathrm{v})$ are as follows:

$$
\mathrm{g}(\mathrm{x}, \mathrm{y})=\left(\frac{1}{2 \pi \sigma_{\mathrm{x}} \sigma_{\mathrm{y}}}\right) \exp \left[-\frac{1}{2}\left(\frac{\mathrm{x}^{2}}{\sigma_{\mathrm{x}}{ }^{2}}+\frac{\mathrm{y}^{2}}{\sigma_{\mathrm{y}}{ }^{2}}\right)+2 \pi \mathrm{jwx}\right]
$$

where " $w$ " is window size where $\sigma_{\mathrm{x}} \sigma_{\mathrm{y}}$ are standard deviation of the input image.

$$
\mathrm{G}(\mathrm{u}, \mathrm{v})=\exp \left\{-\frac{1}{2}\left[\frac{(\mathrm{u}-\mathrm{W})^{2}}{\sigma_{\mathrm{u}}{ }^{2}}+\frac{\mathrm{y}^{2}}{\sigma_{\mathrm{v}}{ }^{2}}+\frac{\mathrm{v}^{2}}{\sigma_{\mathrm{v}}{ }^{2}}\right]\right\}
$$

where $\sigma_{\mathrm{u}}=\frac{1}{2 \pi \sigma_{\mathrm{X}}}$ and $\sigma_{\mathrm{V}}=\frac{1}{2 \pi \sigma \mathrm{y}}$

A set of self-similar functions can be generated from dilation and rotation of the Gabor function $\mathrm{g}(\mathrm{x}, \mathrm{y})$ :

$$
\begin{aligned}
& \mathrm{g}_{\mathrm{mn}}(\mathrm{x}, \mathrm{y})=\mathrm{a}^{-\mathrm{m}} \mathrm{G}(\mathrm{x}, \mathrm{y}) \quad \text { where } \mathrm{a}>1 ; \\
& \mathrm{x}^{\prime}=\mathrm{a}^{-\mathrm{m}}(\mathrm{x} \cos \theta+\mathrm{y} \sin \theta) \quad \mathrm{y}^{\prime}=\mathrm{a}^{-\mathrm{m}}(-\mathrm{x} \sin \theta+\mathrm{y} \cos \theta) \\
& \theta=\mathrm{n} \pi / \mathrm{N} ;
\end{aligned}
$$

$\mathrm{m}=0,1, \ldots, \mathrm{M}-1$ and $\mathrm{n}=0,1, \ldots, \mathrm{N}-1 ; \mathrm{M}$ is the number of scales and $\mathrm{N}$ is the number of orientations. For a given plant image $\mathrm{I}(\mathrm{x}, \mathrm{y})$, the discrete Gabor wavelet transform is given by a convolution as in equation(16):

$$
W_{m n}=\sum_{x i_{i}} \sum_{i i} I\left(x_{1}, y_{1}\right) g_{m n} *\left(x-x_{1}, y-y_{1}\right)
$$

where * indicates the complex conjugate

Applying GF with different orientation and different scale on an image $\mathrm{I}(\mathrm{x}, \mathrm{y})$ with size $\mathrm{P} \times \mathrm{Q}$, we obtain a set of magnitudes:

$$
E(m, n)=\sum_{x} \sum_{y}\left|W_{m n}(x, y)\right|
$$

The standard deviation $\sigma_{m n}$ of the magnitude of the transformed coefficients is computed by the following equation:

$$
\sigma_{\mathrm{mn}}=\sqrt{\frac{\sum_{\mathrm{x} y}\left(\left|\mathrm{~W}_{\mathrm{mn}}(\mathrm{x}, \mathrm{y})\right|-\mu_{\mathrm{mn}}\right)^{2}}{\mathrm{P} \times \mathrm{Q}}} \text { where }_{\mathrm{mn}}=\frac{\mathrm{E}(\mathrm{m}, \mathrm{n})}{\mathrm{P} \times \mathrm{Q}}
$$

The Gabor feature vector is defined by:

$$
f=\left[\sigma_{00}, \sigma_{01}, \ldots, \sigma_{(M-1)(N-1)}\right]
$$

Gabor-Zernike feature for extraction of both texture and shape of the medicinal plant is used. The Gabor-Zernike feature vector is obtained by combining the Gabor and Zernike feature vectors as follows:

1. Normalize the Gabor feature and Zernike feature

2.Gabor-Zernike feature is give by

$$
f_{\text {Gabor-Zernike }}=\left\{f_{\text {Gabor }}\right\} \cup\left\{f_{\text {Zernike }}\right\}
$$

Algorithm 4: Feature extraction using Gabor texture feature:

Input: Original RGB color plant image.

Output: Gabor texture features (Energy values and standard deviation coefficients).

\section{Start:}

Step 1: Read input image, convert it to grayscale and apply preprocess, Obtain the size of image $(\mathrm{P}, \mathrm{Q})$.

Step 2: Calculate the gabor texture values with $\mathrm{m}=0 . .2$ scales and $n=0 . .1$ orientations and its transformed values are obtained.

Step 3: Gabor wavelet transform is obtained with convolving input image with shifted complex conjugate gabor texture values.

Step 4: Energy values and standard deviation coefficients $\left[\sigma_{00}, \sigma_{01}, \sigma_{10}, \sigma_{20}, \sigma_{21}\right]$ are obtained using equation (18).

Stop.

\section{RESUlts AND Discussion}

Retrieval experiment has been carried out on sample of 100 medicinal plants images. The retrieved image is considered as match if and only if it is in the same category as the query image. In addition, the effectiveness of the extracted features has been measured by precision and recall parameters. Precision is the ratio of relevant retrieved images to the total number of retrieved images. Recall is the ratio of relevant images retrieved to the total number of images in the database. The medicinal plant with number 69(Hibiscus Cannabinus) was selected as a query image.

Fig. 6 shows the feature vectors using fourier descriptor, centroid values and gabor texture feature for 10 medicinal plants. The zernike feature values are depicted in Fig. 7. From the experimental results it shows that of the 11 Zernike features only F3, F4, F5, F6, F9 and F10 are varying and are significant for characterizing the medicinal plant shape. The indexing of the testing database was carried out by calculating the Euclidean distance between the feature vectors of query image of all above feature values with database image. The retrieval results are ranked in sorted order with Euclidean distance as shown in Fig. 9(a) to 9(d). Fig. 8 shows the precision and recall rate graph.. It is revealed that gabor and zernike features retrieved more relevant images than the fourier and generic fourier decriptor. The 
retrieval precision is higher with $86 \%$ and lower for combined descriptor with $50 \%$ of retrieval precision.Further, it is clear from Fig. 10(a) that retrieval performance using FDs centroid distance has highest performance than that of other methods. It has a nicely smooth degradation of precision, showing its robust in terms of precision and recall. Centroid distance captures both local and global features of plant and is information preserving. Curvature signature is sensitive to noise and distortion. Since curvature signature requires intensive boundary approximation to make it reliable. Hence retrieval accuracy is not effective with curvature signature. The comparison results are shown in Fig 10(a)-10(e), from which we found that gabor zernike descriptor gives high retrieval performance. Gabor-Zernike deals successfully with scaled and rotated views of images.

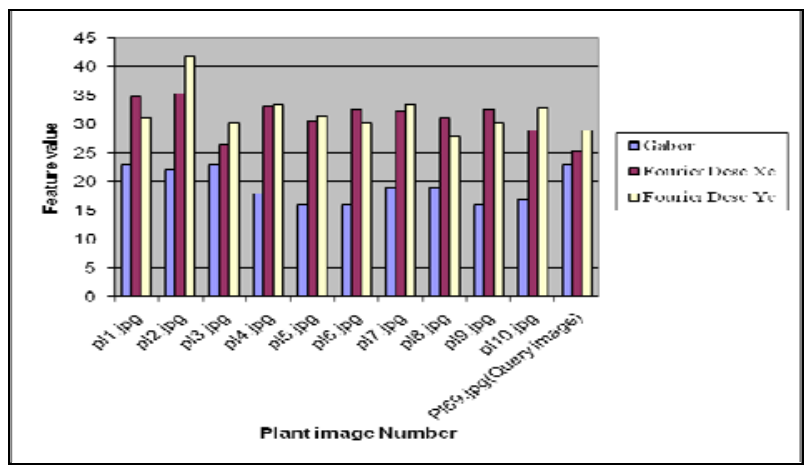

Fig. 6. Gabor and Fourier feature values

Comparision of Zernike Feature Values

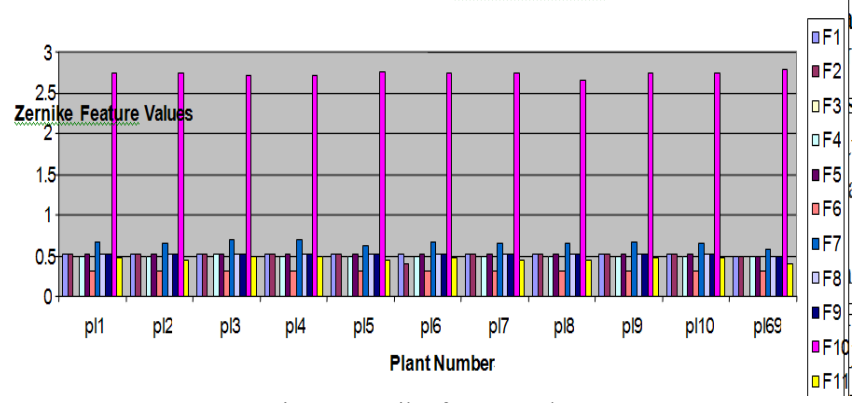

Fig. 7. Zernike feature values

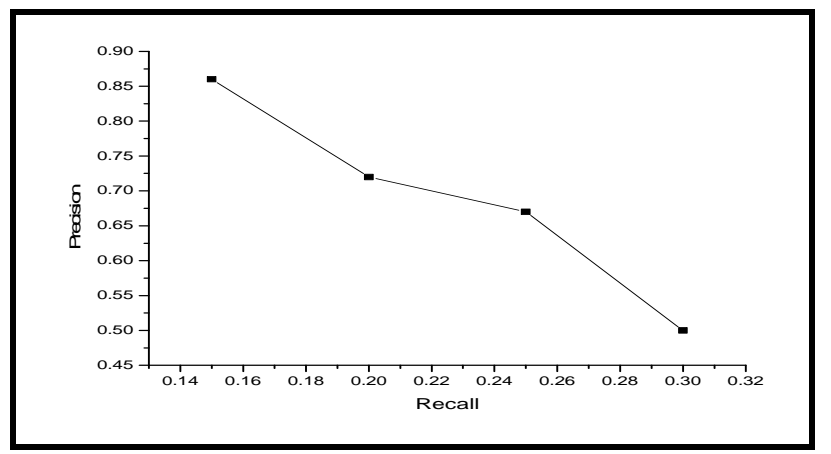

Fig. 8. Precision and recall rate

Because these images produce perspective deformations as a result spatial distribution will be changed substantially. But Gabor-zernike can cope with this type of situation by examining both shape and texture features in all directions. The retrieval results of GFD is also effective but less than gabor-zernike,since GFD captures shape features in both radial and circular directions, the retrieved shapes are more perceptually acceptable. For example, in Fig. 9(b), GFD not only retrieves those similar shapes to the query, but also retrieves perceptually relevant rotated images.

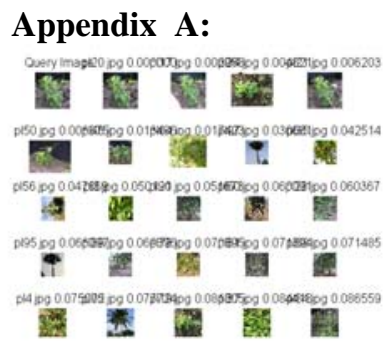

(a)

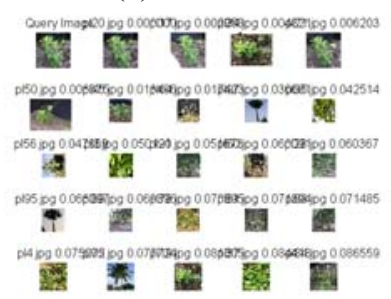

(c)

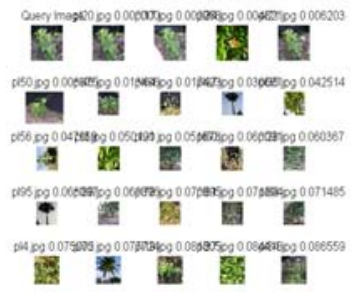

(b)

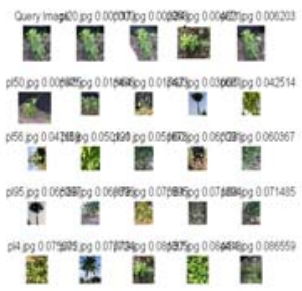

(d)
Fig. 9. Retrieval Using (a) Gabor Zernike (b)Generic Fourier descriptor (c)Fourier descriptor(d) Combination of all descriptor.
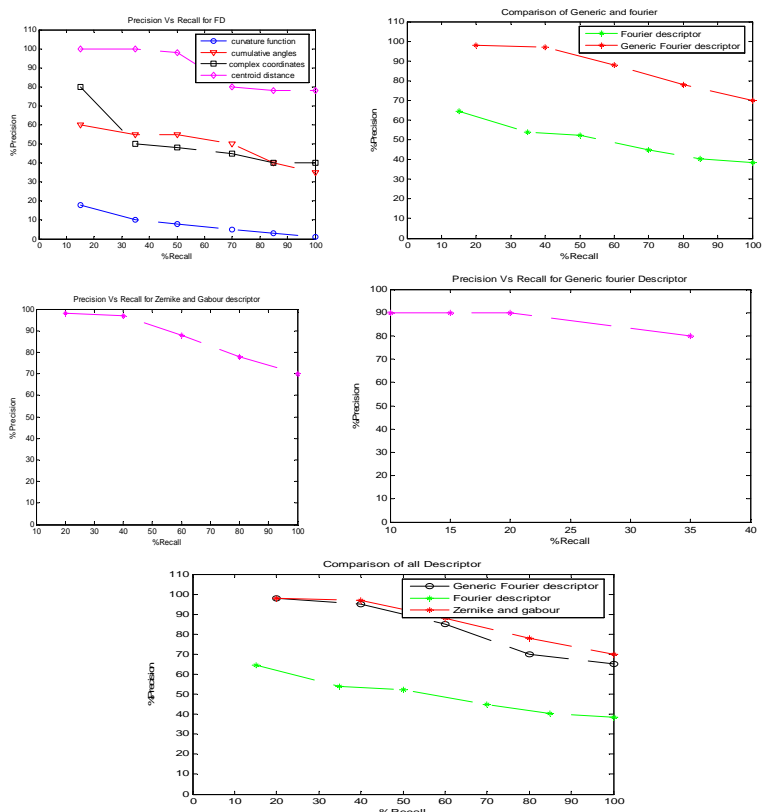

Fig. 10. Comparision of Precision-recall rate of (a) Fourier descriptor (b) Fourier-GFD (c) Zernike and gabor descriptor (d) Generic- fourier descriptor (e) Comparison of all descriptor

\section{CONCLUSION}

We have used different shape and texture features in our Content-Based Image Retrieval (CBIR) system. For shape descriptors we have used fourier, generic fourier descriptor, and zernike features.Gabor filters have been used for texture descriptors. Experimental results showed that the Gabor -zernike descriptor show high retrieval efficiency of $92 \%$ compared to fourier and generic fourier features.

In general, the effective implement ation of a CBIR system is difficult. This is due to the fact that the implementation depends mainly on image and machine vision algorithms. Experimental results showed that use of a single descriptor in 
CBIR systems is not sufficient. Hence, Gabor-Zernike which combines both texture and shape features gives better retrieval efficiency.An approach to improve the precision and recall rate for image retrieval, combine multiple heterogeneous descriptors. As a future work we try to compare our CBIR systems with other features so that medicinal plants are retrieved with respect to different classes.

\section{REFERENCES}

[1] A. Sajjanhar1, Guojun Lu, and Dengsheng Zhang, "Image Retrieval Using Modified Generic Fourier Descriptors," Proceedings of the 19th International Conference on Speech Communication Association (ISCA), pp.1-4, 2004.

[2] A. Khotanzad and Y. H. Hongs, "Invarient imagerecognition by zernike moments," IEEE Transactions on Pattern Analysis and Machine Intelligence, vol. 12, no. 5, pp. 489-497, 1990.

[3] Andre Folkers and Hanan Samet, "Content-based Image Retrieval Using Fourier Descriptors on a Logo Database," Proceedings of the 16th International Conference on Pattern Recognition, vol. 3, pp. 521-524, 2002.

[4] Dengsheng Zhang and Guojun Lu, "A Comparative Study of Fourier Descriptors for Shape Representation and Retrieval," The 5th Asian Conference on Computer Vision, Australia, pp. 1-6, 2002.

[5] Dengsheng Zhang and Guojun Lu, "A Comparative Study on Shape Retrieval Using Fourier Descriptors with Different Shape Signatures," IEEE Transactions on Systems, Man and Cybernetics, vol. 7, no. 3, pp.170-179, 1977.
[6] Dengsheng Zhang and Guojun Lu, "Generic Fourier Descriptor for Shape-based Image retrieval," IEEE Int. Conf. On Multimedia and Expo, PP.1-4, 2002.

[7] Dengsheng Zhang, Aylwin Wong, Maria Indrawan, and Guojun Lu, "Content-based Image Retrieval Using Gabor Texture Features," First IEEE Pacific-Rim Conference on Multimedia, pp. 392-395, 2000.

[8] D. Zhang and G. Lu, "A Comparative Study of Curvature Scale Space and Fourier Descriptors for Shape-Based image Retrieval," Journal of Visual Communication and Image Representation, vol. 14, no.1, pp. 41-60, 2003.

[9] Farzin Mokhtarian and Riku Suomela, "Curvature Scale Space for Robust Image Corner Detection," IEEE Transactions On PAMI, vol. 17, no. 5,1995 .

[10] Gang Zhang, Z.M. Ma, and Qiang Tong, "Shape Feature Extraction Using Fourier Descriptors with Brightness in Content-based Medical Image Retrieval," International Conference on Intelligent Information Hiding and Multimedia Signal Processing, pp. 71-74, 2008.

[11] I. Kunttu, L. Lepistö, J. M. Rauhamaa and A. Visa, "Multiscale Fourier Descriptor for Shape-Based Image Retrieval," Proceedings of the 17th International Conference on Pattern Recognition 1996; vol. 5, no.1, pp. $56-70$.

[12] I. Kunttu, L. Lepistö, J. Rauhamaa and Ari Visa, "Multiscale Fourier Descriptor for Shape Classification," 12th International Conference on Image Analysis and Processing. pp. 319-337, 2003.

[13] T. Andrysiak and Michał Choras, "Image Retrieval Based On Hierarchical Gabor Filters," International Journal of Application, Mathamatics, Computer science, vol. 15, no. 4, pp. 471-480, 2005.

[14] X. Fu, Y. Li, R. Harrison, and S. Belkasim, "Content-Based image Retrieval Using Gabor-Zernike Features," The 18th International Conference on Pattern Recognition(ICPR'06), pp.2-8, 2006.

[15] Ye Bin and Peng Jia-xiong, "Improvement and Invariance Analysis of Zernike Moments using as a Region based Shape Descriptor," Proceedings of the 15th Brazilian Symposium on Computer Graphics and Image Processing, pp.120-126, 2002. 